\title{
REVIEW ARTICLE \\ LAPLACE ADOMIAN DECOMPOSITION METHOD FOR SOLVING A MODEL OF CHRONIC MYELOGENOUS LEUKEMIA (CML) AND T CELL ASSOCIATION
}

\author{
Faiz Alam \\ Department of Mathematics, University of Malakand, Dir(L), Khyber Pakhtunkhwa, Pakistan \\ *Corresponding Author Email: ufaizalam22@gmail.com
}

This is an open access article distributed under the Creative Commons Attribution License, which permits unrestricted use, distribution, and reproduction in any medium, provided the original work is properly cited.

\section{ARTICLE DETAILS}

\section{Article History:}

Received 10 August 2019 Accepted 15 September 2019 Available online 30 October 2019

\section{ABSTRACT}

In this article, it is our purpose that we examine as well as analyze Chronic Myelogenous Leukemia (CML) a mathematical model, a white blood cells cancer. This model shows the association between naive T cells, effector T cells and CML cancer cells in the body, using a system of differential equations which give the rate of change of these three-cell population. We implement a Laplace Adomian Decomposition Method to compute an approximate solution of the considered model. We try to obtain analytic solution for CML model in the form of series that rapidly converges. Further, we also provide some result and stability of the propose model.

\section{KEYWORDS}

Laplace Adomian Decomposition Method, Linear differential equation, Chronic Myelogenous Leukemia (CML) and T Cell Association, Analytical solution.

\section{INTRODUCTION}

Chronic Myelogenous Leukemia (CML) is a type of white blood cell cancer, in which excessively wide range of white blood cells are produce by bone marrow. CML is a disease, in blood system it affects cell circulating. It is also called Chronic granulocytic Leukemia is a steadily developing bone marrow blood and disease that commonly occurs after or during grown up stage and scarcely happen in young human being. The disorder found in more than 1 out of 100,000 people per year worldwide which makes 15 percent approximately of the entire Leukemia in mature human being. In this type of cancer mass are produce in blood. By means of this, cell tends to the myeloid stem cell lineage and because this is a Chronic Leukemia, the cell that we see tend to be more mature type of blood cell in blood stream as opposed to an acute Leukemia, where in the blood stream individual have more immature cells [1].

Chronic Myelogenous Leukemia occurs, that is when a gene that sits on chromosomes 22, which is called BCR gene. The BCR gene is shuffled so that it sits next to a gene on chromosomes 9 that is called the ABL or the ABL gene, a very specific mutation happens. The defect is known as translocation, so we write translocation of a piece of chromosome 9 onto chromosomes 22 that for the purposes of drawing here will look something like this where the BCR gene sits on the ABL gene and they are attached together [2]. The new protein is a type of receptor tyrosine kinase which is constantly on telling cells that they should keep on dividing which is the whole logic of cancer. Individuals are making more cells than they need. In fact, this translocation that puts a piece of chromosomes 9 on chromosome 22 a new type of chromosome produces altogether which is called the "Philadelphia chromosome" named for university it was discovered at. But the problem here is that now we are going to produce a whole bunch of White blood cells we do not need. We might recall one thing that produced here is something that called a Pluripotent meaning that something that has potency or the ability to make plural or many things hematopoietic meaning related to a blood stem cell.

That is a pluripotent hematopoietic stem cell. Which produce two different lineages? As there are a variety of things that could be produced here, but we recall it these types of cells right here these white blood cells that we will see being produced excessively in CML [3]. Because the defect is really high up like around here is where the mutation occurs. We will tend to see an irregular or an unusual amount of platelets or even RBC as well. That translates into a couple of key signs and symptoms. Now CML as it sometimes referred to has three different phases that have distinct symptoms and signs. The first one is the chronic phase. About 90 percent are asymptomatic patients in chronic phase that is without any symptoms. But when they are diagnosed, that is asymptotic individual can see some symptoms and signs that is abdominal fullness, because of the fact that excessive platelets or other WBC which are being made, go to individual spleen to get processed or even destroyed if there is excessively many defective ones causing the spleen to become large which is called Splenomegaly. Splenomegaly and the same thing can happen to individual liver, as individual has a higher Basel metabolism because of all of the extra cells in an individual blood stream. When the liver gets big it is called hepatomegaly, so in a total individual could have Hepatic-Splenomegaly.

A common symptom is having a fever other than the abdominal fullness and this is mainly because of having a large number or a white blood cell count because as mentioned above that means individual basal metabolism has increased, which is just metabolism at rest. The chronic phase will progress, and the next step would be accelerated phase. In this phase individual has more rapidly producing cells and usually these cells are defective therefore it is called accelerated phase [4]. Those that don't actually work properly and this is well illustrated when individual consider the platelets because these patients can bleeding and it is due to platelets are supposed to clot and make sure it can't bleed when cut, it will bleed more when platelets are not working, and these can obvious as petechial, which are just small dots that we might see, which is bleeding from vessels or it is called ecchymosis. Ecchymosis which are just bruising or bruises that occur when even just bump into things very lightly and so have accumulation of blood under the skin that will look like hit it there much intensely than actually had, in the accelerated phase we could also get a fever but this time it is more likely due to opportunistic infections.

This just means that microbes like bacteria or fungi or viruses see in an opportunity to infect a human being or host because the white blood cells are not functioning correctly in the accelerated phase. Lastly, the more advanced phase which someone can be referred to as or the blast or even that the blast crises. This phase is described by fast production of immature cell, which can cause to have some significant bone pain. In bone marrow the production of myoblast increased is related.to bone pain Individual can also have a fever and so as for the reasons we have 
mentioned above either from an opportunistic infection or large number of these cells present in the blood stream. Our model consists of three nonlinear equations $[1,5]$ :

$$
\begin{gathered}
\frac{d T}{d t}=S_{n}-d_{n} \mathrm{~T}-k_{n} \mathrm{~T}\left(\frac{C}{C+\eta}\right) \\
\frac{d E}{d t}=\alpha_{n} k_{n} \mathrm{~T}\left(\frac{C}{C+\mathrm{\eta}}\right)+\alpha \mathrm{eE}\left(\frac{C}{C+\mathrm{\eta}}\right)-d_{e} \mathrm{E}-\gamma \mathrm{eCE} \\
\frac{d C}{d t}=r_{e} \mathrm{C} \ln \left(\frac{C \max }{C}\right)-d_{e} \mathrm{C}-\gamma \mathrm{eCE}
\end{gathered}
$$

Each in one equation shows the rate of change with respect to time with the initial conditions. $T(0)=a_{1}, E(0)=a_{2}, C(0)=a_{3}$.

\section{BASIC CONCEPT OF LAPLACE ADOMIAN DECOMPOSITION METHOD}

Nearly all events that make an appearance in practical life can be narrated with the help of nonlinear ordinary and partial differential equations and in certain instance by integral differential equations [6]. Still nearly all of the mathematical techniques that spread are only able for solving linear differential equations [7-9,11]. A researchers in the 1980's introduced a powerful technique to solve nonlinear differential equations. So, this technique is called the Adomian Decomposition method (ADM). The technique is based on the decomposition of solution of non-linear differential equations in a series of function. Every term of the series is pick up from a polynomial produced by the expansion of power series of an analytic function. In an abstract formulation the Adomian method is so easy but calculating polynomials complexity appeared which a non-trivial job becomes. This technique has been globally utilized for solving equations that comes from nonlinear methods and solve fractional differential equations. Consider the following equation.

$L U+N U=g(t)$

Where $N$ is nonlinear operator and $L$ is linear operator and $f(t)$ is analytic function.

\subsection{The ADM collaborated with Laplace Transform}

The Laplace transform $L$ is an integral transform introduced by Pierre Simon Laplace and is a powerful and very effective toll for solving partial and ordinary differential equations which transform to an elementary algebraic equation from the actual differential equation. Before using Laplace transform collaborate with ADM we recall some fundamental definitions and results [9].

\subsubsection{Definition}

Stated a function $g(t)$ defined for all $t \geq 0$ the Laplace transform of $\mathrm{g}$ is the function $\mathrm{G}$ defined by:

$L[g(t)]=\int_{0}^{\infty} g(t) e^{-s t} d t$.

The improper integral converges for the entire values of $s$. In particular $L\left[t^{q}\right]=\frac{q !}{s^{q}+1}$. It is already familiar that there exists a bijection between sets of a functions fulfilling some postulate and the set of their Laplace transforms. Therefore, it is natural to define the inverse Laplace transform of $G(s)$.

\subsubsection{Definition}

Stated a continuous function $\mathrm{g}(\mathrm{t})$, if $G(s)=L[g(t)]$ then $g(t)$ is the called the inverse Laplace transform of $G(s)$ and is denoted by $g(t)=$ $L^{-1}[G(s)]$. The Laplace transform has derivative properties;

$$
\begin{gathered}
L\left[g^{q}(t)\right]=s^{q} L[g(t)]-\sum_{k=0}^{q-1} s^{q-1-k} g^{k}(0) \\
L\left[t^{q} g(t)\right]=(-1)^{q} G^{q}(s),
\end{gathered}
$$

where the superscript $n$ denotes $q$ th derivative with respect to $t$ for $g(t)$ and with respect to $\mathrm{s}$ for $G q(s)$. Applying Laplace transform to equation (2)

$$
L[L U]+L[N U]=L[f(t)]
$$

$$
\begin{gathered}
s L[U]-U(0)+L[N U]=L[f(t)] \\
s L[U]=U(0)-L[N U]+L[f(t)] \\
L[U]=\frac{U(0)}{s}-\frac{1}{s} L[N U]+\frac{1}{s} L[f(t)] .
\end{gathered}
$$

Now applying Laplace inverse transform $[6,9]$.

$$
\begin{aligned}
L^{-1} L[U] & =L^{-1}\left[\frac{U(0)}{s}\right]-L^{-1}\left[\frac{1}{s} L(N U)\right]+L^{-1}\left[\frac{1}{S} L f(t)\right] \\
U(t) & \left.=L^{-1} U(0)+L^{-1}\left[\frac{1}{S} L f(t)\right]-L^{-1}\left[\frac{1}{S}\right] L[N U]\right] .
\end{aligned}
$$

Now the Adomian Decomposition Method indicate the infinite series of an unknown function $\mathrm{U}$ of the form

$U=\sum_{n=0}^{\infty} U n$

Or equals to

$U=U_{0}+U_{1}+U_{2}$

where the elements $U(n)$ will be resolute returning. Additionally, the method defines the non-linear term by Adomian polynomials. More literally, the ADM assumes that $N(u)$ the nonlinear operator can be decomposed by a series of polynomials given by $[6,9]$ :

$N(U)=\sum_{n=0}^{\infty} A_{n} U_{n}$

Plug equations (3), (4) and (5) in (2)

$$
\begin{aligned}
& \sum_{n=0}^{\infty} U n(t)=L[U(0)]+L^{-1}\left[\frac{1}{s} L[f(t)]-L^{-1}\left[\frac{1}{s} L \sum_{n=0}^{\infty} A n U n\right]\right] \\
& U_{0}(t)+U_{1}(\mathrm{t})+\cdots=\mathrm{L}[\mathrm{U}(0)]+L^{-1}\left[\frac { 1 } { S } \mathrm { L } \left[\mathrm { f } ( \mathrm { t } ) 1 \left[\frac{1}{S} \mathrm{~L}\left[A_{0} U_{0}\right]+\frac{1}{S} \mathrm{~L}\left[A_{1} U_{1}\right.\right.\right.\right. \\
& +\frac{1}{S}\left[A_{2} U_{2}\right]+\ldots
\end{aligned}
$$

Therefore, the proper reappearance result could be defined by

$$
\begin{gathered}
U_{0}(t)=L^{-1}[\mathrm{U}(0)]-L^{-1}\left[\frac{1}{S} \mathrm{~L}[\mathrm{f}(\mathrm{t})]\right. \\
U_{1}(t)=L^{-1}\left[\frac{1}{S} \mathrm{~L}\left[A_{0} U_{0}\right]\right. \\
U_{2}(t)=L^{-1}\left[\frac{1}{S} \mathrm{~L}\left[A_{1} U_{1}\right]\right. \\
U_{n}(t)=L^{-1}\left[\frac{1}{S} \mathrm{~L}\left[A_{n} U_{n}\right] .\right.
\end{gathered}
$$

Now let us take

$U(x)=\sum_{n=0}^{\infty} U_{n}$,

$N(u)=\sum_{n=0}^{\infty} A_{n}$

where $A(n)$ are Adomian polynomial. To compute $A n$ take $N(u)=g(u)$ to be a nonlinear function in $U$. where $U=U(x)$ and consider the Taylor series expansion of $g(u)$ around $U_{0}$.

$$
\begin{aligned}
& g(U)=\mathrm{g}\left(U_{0}\right)+\mathrm{g}^{\prime}\left(U_{0}\right)\left(\mathrm{U}-U_{0}\right)+\frac{1}{2 !} \mathrm{g} \prime\left(U_{0}\right)\left(U-U_{0}\right)^{2}+ \\
& \frac{1}{3 !} \mathrm{g}{ }^{\prime \prime \prime}\left(U_{0}\right)\left(U-U_{0}\right)^{3} \cdots \\
& \text { But } U=U_{0}+U_{1}+U_{2}+\cdots \\
& g(U)=\mathrm{g}\left(U_{0}\right)+\mathrm{g}^{\prime}\left(U_{0}\right)+\frac{1}{2 !} \mathrm{g}{ }^{\prime \prime}\left(U_{0}\right)\left(U_{1}+U_{2+\cdots}\right)^{2} \\
& \quad+\frac{1}{3 !} \mathrm{g}{ }^{\prime \prime}\left(U_{0}\right)\left(U_{1}+U_{2}+\cdots\right)^{3}
\end{aligned}
$$

Now to expand Equation (6) To pick up the Adomian polynomials we wish early to recorder and reorganize the terms. Indeed, one wish to find the order of every term in Equation (6) which really depends on a couple, that is the subscripts and the power of the $U n$. For instance, $U_{1}$ is of order 1 , $U_{1}^{2}$ is order $2, U_{2}^{3}$ is of order 6 and so on and general $U n^{K}$ is of order $K_{n}$. In case a specific term connects to the multiplication of $U_{n}$. In every term Its order can be determined by the sum of the terms of the $U_{n}$. That is $U_{2}^{3} U_{1}^{2}$ is 
of order 8 since $(3)(2)+(2)(1)=8$. As a result, rearrange the terms in the expansion Equation (6) according to the order we have

$$
\begin{aligned}
g(U)=g\left(U_{0}\right)+\mathrm{g}^{\prime}( & \left.U_{0}\right) U_{1}+\mathrm{g}^{\prime}\left(U_{0}\right) U_{0} \frac{1}{2 !} \mathrm{g}{ }^{\prime \prime}\left(U_{0}\right)\left(U_{1}\right)^{2}+\mathrm{g}^{\prime}\left(U_{0}\right) U_{3} \\
& +\frac{2}{2 !} \mathrm{g}^{\prime \prime}\left(U_{0}\right) U_{1} U_{2}+\frac{1}{3 !} \mathrm{g}^{\prime \prime}\left(U_{0}\right)\left(U_{1}\right)^{3} \\
& +\mathrm{g}^{\prime}\left(U_{0}\right) U_{3} \frac{1}{2 !} \mathrm{g}{ }^{\prime \prime}\left(U_{0}\right)\left(U_{0}\right)^{2}+\frac{2}{2 !} \mathrm{g}^{\prime \prime}\left(U_{0}\right) U_{1} U_{3} \\
& +\frac{3}{3 !} \mathrm{g}{ }^{\prime \prime}\left(U_{0}\right)\left(U_{1}\right)^{2} U_{2}+\cdots
\end{aligned}
$$

The Adomian polynomials are build up in a many approach so that the polynomial $A_{1}$ consist of all term in the expansion Equation (7) of order 1, $A_{2}$ consist of all terms of order 2 and so on. In general, $A n$ consist of all terms of order $n$. Therefore, Adomian polynomials of first five terms are listed below

$$
\begin{gathered}
A_{0}=\mathrm{g}\left(U_{0}\right) \\
A_{1}=\mathrm{g}^{\prime}\left(U_{0}\right) U_{1} \\
A_{2}=\mathrm{g}^{\prime}\left(U_{0}\right) U_{2}+\frac{1}{2 !} \mathrm{g}^{\prime \prime}\left(U_{0}\right) U_{1}^{2} \\
A_{3}=\mathrm{g}^{\prime}\left(U_{0}\right) U_{3}+\frac{2}{2 !} \mathrm{g}^{\prime \prime}\left(U_{0}\right) U_{1} U_{2}+\frac{1}{3 !} \mathrm{g}^{\prime \prime \prime}\left(U_{0}\right) U_{1}^{3} \\
A_{4}=\mathrm{g}^{\prime}\left(U_{0}\right) U_{4}+\frac{1}{2 !} \mathrm{g}{ }^{\prime \prime}\left(U_{0}\right)\left(2 U_{1} U_{3}+U_{2}^{2}\right)+\frac{3}{3 !} \mathrm{g}^{\prime \prime}\left(U_{0}\right) U_{1}^{2} U_{2}+
\end{gathered}
$$$$
\frac{1}{4 !} \mathrm{g} \cdot{ }^{\prime \prime \prime}\left(U_{0}\right) U_{1}^{4}
$$

The Adomian polynomial $A_{n}$ was first popularized by Adomian himself; it was defined via the general formula

$$
A_{n}\left(U_{0}, U_{1} U_{2} \ldots\right)=\frac{1}{n !} \frac{d^{n}}{d \lambda^{n}}\left[N\left(\sum_{n=0}^{\infty} U_{k} \cdot \lambda^{k}\right)\right] \lambda=0
$$

To find the $A_{n}$ by Adomian general formula these polynomials will be computed as follow

$$
\begin{gathered}
A_{0}=N\left(U_{0}\right) \\
A_{1}=\frac{d}{d \lambda} N\left(U_{0}+U_{1} \lambda\right)_{\lambda=0}=N\left(U_{0}\right) U_{1} \\
A_{2}=\frac{1}{2 !} \frac{d 2}{d \lambda 2}\left(U_{0}+2 U_{2} \lambda\right) N^{\prime \prime}\left(U_{0}+U_{1} \lambda\right)_{\lambda=0} \\
=N^{\prime}\left(U_{0}\right) U_{2}+\frac{1}{2 !} N^{\prime \prime}\left(U_{0}\right) U_{1}^{2}
\end{gathered}
$$

\subsection{Solving CML Model using LADM}

In this section we apply LADM to our three nonlinear equations that is eq (1)

$L\left[\frac{d T}{d t}\right]=\mathrm{L}\left[S_{n}-d_{n} \mathrm{~T}-K_{n} \mathrm{~T}\left(\frac{C}{C+\eta}\right)\right]$

$L\left[\frac{d E}{d t}\right]=\mathrm{L}\left[K_{n} \mathrm{~T}\left(\frac{C}{C+\eta}\right)+\alpha_{e} \mathrm{E}\left(\frac{C}{C+\eta}\right)-d_{e} \mathrm{E}-\gamma_{e} \mathrm{CE}\right]$

$L\left[\frac{d C}{d t}\right]=\mathrm{L}\left[\gamma_{e} \mathrm{Cln}\left(\frac{C \max }{C}\right)-d_{e} \mathrm{C}-\gamma_{e} \mathrm{CE}\right]$.

Apply the formulas for Laplace

$\left.\mathrm{L}[\mathrm{T}]-\mathrm{T}(0)=\mathrm{L}\left[\mathrm{S}_{\mathrm{n}}\right]-\mathrm{L}\left[\mathrm{d}_{\mathrm{n}} \mathrm{T}\right]-\mathrm{L}\left[\mathrm{K}_{\mathrm{n}} \mathrm{T}\right]\left(\frac{\mathrm{C}}{\mathrm{C}+\mathrm{n}}\right)\right]$

$L[E]-E(0)=L\left[\alpha_{n} K_{n}\left(\frac{C}{C+\eta}\right)\right]+L\left[\alpha_{e} E\left(\frac{C}{C+\eta}\right)\right]-L\left[d_{e} E\right]-L\left[\gamma_{e} C E\right]$

$L[C]-C(0)=L\left[\gamma_{e} C \ln \left(C_{\max }\right)-L\left[\gamma_{e} C \ln (C)\right]-L\left[d_{e} C\right]-L\left[\gamma_{e} C E\right]\right.$.

Using the initial conditions

$L[T]=a_{1}+\frac{S_{n}}{n}-d_{n} \mathrm{~L}[\mathrm{~T}]-K_{n} \mathrm{~L}\left[\mathrm{~T}\left(\frac{C}{C+\mathrm{\eta}}\right)\right]$

$L[E]=a_{2}+\alpha_{n} K_{n} L\left[T\left(\frac{C}{C+\eta}\right)\right]+\alpha_{e} L\left[E\left(\frac{C}{C+\eta}\right)\right]-d_{e} L[E]-\gamma_{c} L[C E]$
$L[C]=a_{3}+r_{e} \ln \left(C_{\max }\right) L[C]-r_{e} L[C \ln (C)]-d_{e} L(C)-\gamma_{e} L[C E]$.

Let

$A=\frac{T C}{\mathrm{C}+\mathrm{\eta}}, B=\frac{C E}{C+\mathrm{\eta}}, D=C E$ and $M=C \ln C$, where $T, E$ and $C$ are the linear operator so

$T=\sum_{n=0}^{\infty} T_{n}, E=\sum_{n=0}^{\infty} E_{n}$ and $C=\sum_{n=0}^{\infty} C_{n}$.

The nonlinear operator $A, B, D$ and $M$ are

$A=\sum_{n=0}^{\infty} A_{n}, B=\sum_{n=0}^{\infty} B_{n}, D=\sum_{n=0}^{\infty} D_{n}$ and $M=\sum_{n=0}^{\infty} M_{n}$,

Where $A_{n}, B_{n}, D_{n}$ and $M_{n}$ are the Adomian polynomials and are given by

$A_{0}=\frac{C_{0} T_{0}}{C_{0}+\eta}$

$A_{1}=\frac{C_{0}^{2} T_{1}+\eta C_{0} T_{1}+\eta C_{1} T_{0}}{\left(C_{0}+\eta\right)^{2}}$

$A_{2}=\frac{C_{1}^{2} C_{0} T_{0}}{\left(C_{0}+\eta\right)^{3}}-\frac{C_{1}^{2} T_{0}}{\left(C_{0}+\eta\right)^{2}}+\frac{C_{2} C_{0} T_{0}}{\left(C_{0}+\eta\right)^{2}}-\frac{C_{2} T_{0}}{C_{0}+\eta}-\frac{C_{2} C_{0} T_{0}}{\left(C_{0}+\eta\right)^{2}}$

$$
+\frac{C_{1} T_{1}}{C_{0}+\eta}+\frac{C_{0} T_{2}}{C_{0}+\eta}
$$

$$
\begin{aligned}
& B_{0}=\frac{C_{0} E_{0}}{C_{0}+\eta} \\
& B_{1}=\frac{C_{0}^{2} E_{1}+\eta C_{0} E_{1}+}{\left(C_{0}+\eta\right)^{2}} \\
& \mathrm{~B}_{2}=\frac{\mathrm{C}_{1}^{2} \mathrm{C}_{0} \mathrm{E}_{0}}{\left(\mathrm{C}_{0}+\eta\right)^{3}}-\frac{\mathrm{C}_{1}^{2} \mathrm{E}_{0}}{\left(\mathrm{C}_{0}+\eta\right)^{2}} \\
& . \\
& . \\
& D_{0}=C_{0} E_{0} \\
& \mathrm{D}_{1}=\mathrm{C}_{1} \mathrm{E}_{0}+\mathrm{C}_{0} \mathrm{E}_{1} \\
& D_{2}=C_{2} E_{0}+C_{1} E_{1}+C_{0} E_{2} \\
& . \\
& \cdot \\
& M_{0}=C_{0} \ln C_{0} \\
& M_{1}=C_{1}+C_{1} \ln C_{0} \\
& 2 C_{1}^{2}
\end{aligned}
$$$$
B_{1}=\frac{C_{0}^{2} E_{1}+\eta C_{0} E_{1}+\eta C_{1} E_{0}}{\left(C_{0}+\eta\right)^{2}}
$$$$
B_{2}=\frac{C_{1}^{2} C_{0} E_{0}}{\left(C_{0}+\eta\right)^{3}}-\frac{C_{1}^{2} E_{0}}{\left(C_{0}+\eta\right)^{2}}+\frac{C_{2} C_{0} E_{0}}{\left(C_{0}+\eta\right)^{2}}-\frac{C_{2} E_{0}}{\left(C_{0}+\eta\right)}-\frac{C_{2} C_{0} E_{0}}{\left(C_{0}+\eta\right)^{2}}
$$$$
+\frac{\mathrm{C}_{1} \mathrm{E}_{1}}{\mathrm{C}_{0}+\eta}+\frac{\mathrm{C}_{0} \mathrm{E}_{2}}{\mathrm{C}_{0}+\eta}
$$

Applying the formula for Laplace

$$
\begin{gathered}
L\left[\sum_{n=0}^{\infty} T n\right]=\frac{a_{1}}{s}+\frac{S_{n}}{s^{2}}-\frac{d_{n}}{s} L\left[\sum_{n=0}^{\infty} T n\right]-\frac{K_{n}}{S} L\left[\sum_{n=0}^{\infty} A n\right] \\
L\left[\sum_{n=0}^{\infty} E n\right]=\frac{a_{2}}{S}+\frac{\propto_{n} K_{n}}{S} L\left[\sum_{n=0}^{\infty} A n\right]+\frac{\alpha_{n}}{s} L\left[\sum_{n=0}^{\infty} B n\right]-\frac{d_{e}}{s} L\left[\sum_{n=0}^{\infty} E n\right] \\
-\frac{\gamma_{e}}{S} L\left[\sum_{n=0}^{\infty} D n\right]
\end{gathered}
$$


$L\left[\sum_{n=0}^{\infty} C n\right]=\frac{a_{3}}{S}+\frac{\gamma_{e} \ln \left(C_{\max }\right)}{S} L\left[\sum_{n=0}^{\infty} C n\right]-\frac{\gamma_{e}}{S} L\left[\sum_{n=0}^{\infty} M n\right]$

$$
-\frac{d_{e}}{s} L\left[\sum_{n=0}^{\infty} c-n\right]-\frac{\gamma_{c}}{s} L\left[\sum_{n=0}^{s} D n\right] \text {. }
$$

It results

$$
\begin{aligned}
& L\left[T_{0}\right]=\frac{a_{1}}{s}+\frac{S_{n}}{s^{2}} \\
& L\left[T_{1}\right]=\frac{d_{n}}{s} L\left[T_{0}\right]-\frac{K_{n}}{s} L\left[A_{0}\right] \\
& L\left[T_{2}\right]=-\frac{d_{n}}{s} L\left[T_{1}\right]-\frac{K_{n}}{s} L\left[A_{1}\right]
\end{aligned}
$$

$L\left[T_{n+1}\right]=-\frac{d_{n}}{s} L\left[T_{n}\right]-\frac{K_{n}}{s} L\left[A_{n}\right]$

$L\left[E_{0}\right]=\frac{a_{2}}{s}$

$L\left[E_{1}\right]=\frac{\propto_{n} K_{n}}{s} L\left[A_{0}\right]+\frac{\propto_{e}}{s} L\left[E_{0}\right]-\frac{\gamma_{e}}{s} L\left[D_{0}\right]$

$L\left[E_{2}\right]=\frac{\propto_{n} K_{n}}{s} L\left[A_{1}\right]+\frac{\propto_{e}}{s} L\left[B_{1}\right]-\frac{d_{e}}{s} L\left[E_{1}\right]-\frac{\gamma_{e}}{s} L\left[D_{1}\right]$

$L\left[E_{n+1}\right]=\frac{\alpha_{n} K_{n}}{s} L\left[A_{n}\right]+\frac{\alpha_{n}}{s} L\left[B_{n}\right]-\frac{d_{e}}{s} L\left[E_{n}\right]-\frac{\gamma_{n}}{s} L\left[D_{n}\right]$ (9)

$L\left[C_{0}\right]=\frac{a_{3}}{S}$

$L\left[C_{1}\right]=\frac{\gamma_{c} \ln \left(C_{\max }\right)}{S} L\left[C_{0}\right]-\frac{\gamma_{c}}{S} L\left[M_{0}\right]-\frac{d_{e}}{S} L\left[C_{0}\right]-\frac{\gamma_{c}}{S} L\left[D_{0}\right]$

$L\left[C_{2}\right]=\frac{\gamma_{c} \ln \left(C_{\max }\right)}{S} L\left[C_{1}\right]-\frac{\gamma_{c}}{S} L\left[M_{1}\right]-\frac{\mathrm{d}_{\mathrm{e}}}{\mathrm{S}} \mathrm{L}\left[\mathrm{C}_{1}\right]-\frac{\gamma_{\mathrm{c}}}{\mathrm{S}} \mathrm{L}\left[\mathrm{D}_{1}\right]$

$L\left[C_{n+1}\right]=\frac{\gamma_{c} \ln \left(C_{\max }\right)}{S} L[n]-\frac{\gamma_{c}}{S} L\left[M_{n}\right]-\frac{d_{e}}{S} L\left[C_{n}\right]-\frac{\gamma_{c}}{S} L\left[D_{n}\right]$

\subsection{Application}

In this section, we try some practical examples and present the numerical result in the figure below.

The initial conditions are:

$T(0)=\frac{1512 \text { cell }}{\mu \mathrm{l}}, E(0)=\frac{25 \mathrm{cells}}{\mu \mathrm{l}}$,

$C(0)=\frac{10000 \mathrm{cells}}{\mu \mathrm{l}}$.
Adomian polynomials and inverse Laplace transformations give the solution.

$L\left[T_{n+1}\right]=-\frac{d_{n}}{S} L\left[T_{n}\right]-\frac{K_{n}}{S} L\left[A_{n}\right]$

$L\left[E_{n+1}\right]=\frac{\alpha_{n} K_{n}}{S} L\left[A_{n}\right]+\frac{\alpha_{n}}{S} L\left[B_{n}\right]-\frac{d_{e}}{S} L\left[E_{n}\right]-\frac{\gamma_{n}}{S} L\left[D_{n}\right]$

$L\left[C_{n+1}\right]=\frac{r_{c} \ln \left(C_{\max }\right)}{s} L\left[C_{n}\right]+\frac{r_{n}}{S} L\left[M_{n}\right]-\frac{d_{e}}{s} L\left[C_{n}\right]-\frac{\gamma_{c}}{S} L\left[D_{n}\right]$

We start to iteration with

$T_{0}=1512+S_{n} t$

$T_{1}=\frac{-d_{n}}{s} a_{1} t-\frac{d_{n} S_{n} t^{2}}{2}-K_{n} A_{0}$

$T_{2}=\frac{d_{n}^{2} a_{1} t^{2}}{2}+\frac{d_{n}^{2} S_{n} t^{3}}{6}-\frac{K_{n} d_{n}^{2} t^{2}}{6}-K_{n} A_{1}$

Hence the infinite series solution of the T cells is given by:

$T(t)=T_{0}+T_{1}+T_{2}+\cdots$

$E_{0}=25$

$$
\begin{aligned}
& E_{1}=\alpha_{n} K_{n} A_{0}+\alpha_{e} B_{0}-\alpha_{e} a_{2} t-\gamma_{e} D_{0} \\
& \begin{aligned}
E_{2}=\alpha_{n} K_{n} A_{1}+\alpha_{e} B_{1} & -d_{e} \alpha_{e} K_{n} A_{0} t-d_{e} \alpha_{e} B_{0} t+\frac{d_{e} \alpha_{e} a_{2} t^{2}}{2}-d_{e} \gamma_{e} D_{0} t \\
& -\gamma_{e} D_{1}
\end{aligned}
\end{aligned}
$$

$E(t)=E_{0}+E_{1}+E_{2}+\cdots$

$C_{0}=10000$

$$
\begin{aligned}
& C_{1}=r_{c} \ln \left(C_{\max }\right) a_{3} t- \\
& \begin{aligned}
C_{2}=\frac{r_{c}^{2} \ln \left(C_{\max }\right)^{2} a_{3} t^{2}}{2} & -d_{e} a_{3} t-\gamma_{c} D_{0} \ln \left(C_{\max }\right)^{2} M_{0} t-\frac{r_{c} \ln \left(C_{\max }\right) d_{c} a_{3} t^{2}}{2} \\
& -r_{c}^{2} \ln \left(C_{\max }\right) D_{0} t-r_{c} M_{1}-\frac{r_{c} d_{c} \ln \left(C_{\max }\right) a_{3} t^{2}}{2} \\
& -d_{c} r_{c} M_{0} t-\frac{d_{c}^{2} a_{3} t^{2}}{2}-d_{c} r_{c} D_{0} t-\gamma_{c} D_{1}
\end{aligned}
\end{aligned}
$$

$C(t)=C+C_{1}+C_{2}+\cdots$.

We compare the numerical result with table and plot for first three terms Maple software which gives the following graphical result:

Table 1: Three terms Maple software

\begin{tabular}{|c|c|c|c|c|c|c|c|c|c|c|c|}
\hline$S_{n}$ & $d_{e}$ & $d_{n}$ & $d_{c}$ & $K_{n}$ & $\eta$ & $\alpha_{e}$ & $\alpha_{n}$ & $C_{\max }$ & $r_{c}$ & $\gamma_{e}$ & $\gamma_{c}$ \\
\hline 0.37 & 0.30 & 0.230 & 0.024 & 0.062 & 720 & 0.98 & 0.14 & 230000 & 0.0057 & 0.057 & 0.0034 \\
\hline 0.71 & 0.12 & 0.071 & 0.68 & 0.063 & 43 & 0.53 & 0.56 & 190000 & 0.23 & 0.0077 & 0.047 \\
\hline
\end{tabular}




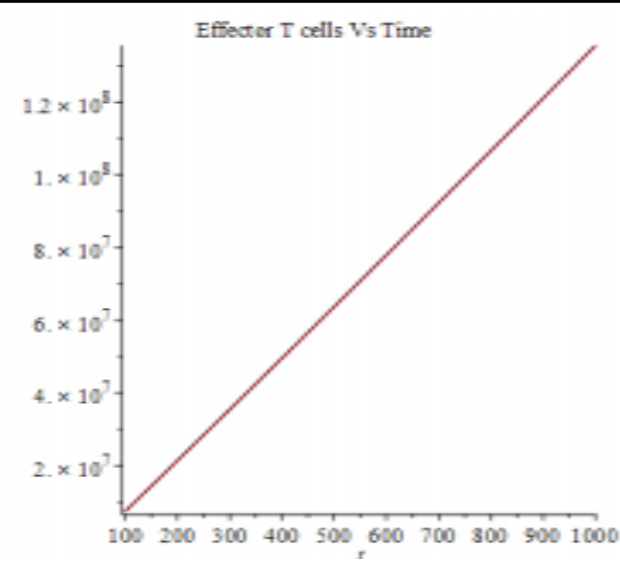

Figure 1: E Cells versus time

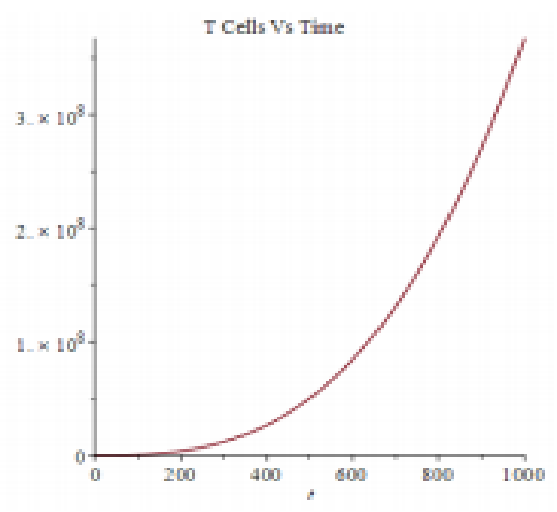

Figure 2: T Cells versus time

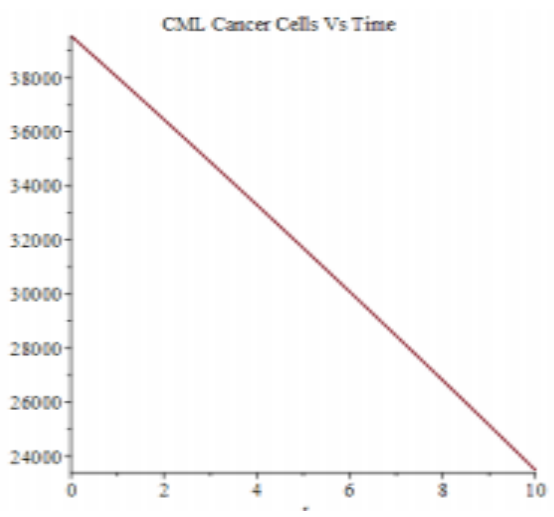

Figure 3: CML Cells versus time

We have successfully established a series solution of Chronic Myelogenous Leukemia CML and T Cell Association that is the Cancer model. For a concerned series solution, we have utilized LADM. Hence, we conclude that LDAM is powerful tools to obtain an approximate or analytical solution to linear as well as nonlinear deferential equations. In the future, we can solve more complicated model by applying LADM.

\section{STABILITY ANALYSIS}

To review the nature of system the deferential equations. We try to pick up equilibrium solution and then linearize around the particular to investigate the stability. To minimize the arithmetic, we clarify early the model by re-scaling the variable.

\subsection{Clarification of the model}

Here we utilize the common re-scaling techniques that are non dimensionalization to cut down the parameter numbers from 12 to 8 . The time and population are rescaled as follow [1]: $T$ is re-scaled by multiplying the factor of $\frac{d_{n}}{s_{n}}$, and $E$ is re-scaled by a factor of $\frac{\gamma_{c}}{d_{n}}, C$ is rescaled by a factor of $\frac{\gamma_{e}}{d_{n}}$ and time $t$ is re-scaled by a factor of $d_{n}$ Equation (1) becomes

$\frac{d T}{d t}=1-T-\frac{K_{n}}{S_{n}} T\left(\frac{C}{C+\frac{\gamma_{e n}}{d_{n}}}\right)$

$$
\begin{aligned}
& \frac{d T}{d t}=\frac{\gamma_{c} \alpha_{n} K_{n} S_{n}}{d_{n}^{3}} T\left(\frac{C}{C+\frac{\gamma_{e \eta}}{d_{n}}}\right)-\frac{\alpha_{e}}{d_{n}} E\left(\frac{C}{C+\frac{\gamma_{e \eta}}{d_{n}}}\right)-\frac{d_{e}}{d_{n}} E-C E \\
& \frac{d T}{d t}=\frac{r_{c}}{d_{n}} C \ln \left(\gamma_{e} C \frac{\max }{\frac{d_{n}}{C}}\right)-\frac{d_{e}}{d_{n}} C-C E
\end{aligned}
$$

Now let define the new coefficients as: $\lambda_{1}=\frac{K_{n}}{d_{n}}, \lambda_{2}=\frac{\gamma_{e} \eta}{d_{n}}, \lambda_{3}=\frac{\alpha_{n} K_{n} S_{n} \gamma_{C}}{d_{n}^{3}}$, $\lambda_{4}=\frac{\alpha_{e}}{d_{n}}, \lambda_{5}=\frac{d_{e}}{d_{n}}, \lambda 6=\frac{r_{c}}{d_{n}}, \lambda_{7}=\frac{\gamma_{e} C_{\max }}{d_{n}}$ and $\lambda_{8}=\frac{d_{c}}{d_{n}}$ so Equation (11) becomes

$\frac{d T}{d t}=1-T-\lambda_{1} T\left(\frac{C}{C+\lambda_{2}}\right)$

$\frac{d T}{d t}=\lambda_{3} T\left(\frac{C}{C+\lambda_{2}}\right)-\lambda_{4} E\left(\frac{C}{C+\lambda_{2}}\right)-\lambda_{5} E-C E$

$\frac{d C}{d t}=\lambda_{6} C \ln \left(\frac{\lambda_{7}}{C}\right)-\lambda_{8} C-C E$

\subsection{Equilibrium Solutions}

Now for sake of equilibrium solution, to determine $T, E$, and $C$ we set each of the carried deferential equations Equation (12) equal to zero and so we got values of $T, E$, and $C$ for which the system will be not any more change, that is entire rate of change or derivatives will be zero. We assume that $C=0$. Then Equation (12) will provide values of $T=1, E=0$, and there will be no one else equilibrium for which $C=0$. We let $E_{1}=(1 ; 0 ; 0)$ show equilibrium solution is trivial (healthy). Now, the value of $C$ for all other equilibrium provided to Equation (12) the following equation received.

$$
0=\lambda_{8}-\lambda_{6} \ln \left(\lambda_{7}\right)+\lambda_{6} \ln (C)+\frac{\lambda_{3} C\left(C+\lambda_{2}\right)}{\left(C+\lambda_{2}+\lambda_{1} C\right)\left[\left(C+\lambda_{2}\right)\left(C+\lambda_{5}\right)-\lambda_{4}\right]}
$$

On the right-hand side of Equation (13) the third term is logarithmic in $C$, and so rises as $C$ rises. $C$ must be negative in order the fourth term, to be zero. Thus, when $C$ is positive, the rational function in fourth term is decreases as $C$ increases, in order to balance the right-hand side of Equation (13) there must be one value of $C$. This aid there is maximum one other equilibrium solution which we indicate by $E_{2}=(T, C, E)$. When $\lambda_{8}<$ $\lambda_{6}$ or when $\frac{d_{c}}{r_{c}}<\ln \left(\frac{\gamma_{c} c_{\max }}{d_{n}}\right)$ this equilibrium solution appears. For the mention equilibrium to be actually purposeful all the three cells population should be non-negative. The appearing condition in Equation (12) are filled trivially. Hence, the equilibrium must be realistic one, and no advanced materials are collected from these conditions.

\subsection{Linearization of the system}

To deduce the nature of cells population close to every one of the equilibrium solutions we wish to evaluate the linearization of the system, which we pick from Jacobian matrix of the above system. The Jacobian matrix of Equation (11) is

$J=\left[\begin{array}{ccc}-1-\lambda_{1}\left(\frac{C}{C+\lambda_{2}}\right) & 0 & -\lambda_{1} T \frac{\lambda_{2}}{\left(C+\lambda_{2}\right)^{2}} \\ \lambda_{3}\left(\frac{C}{C+\lambda_{2}}\right) & \lambda_{4}\left(\frac{C}{C+\lambda_{2}}\right)-\lambda_{5}-C & \lambda_{3} T \frac{C}{\left(C+\lambda_{2}\right)^{2}}+-\lambda_{4} E \frac{C}{\left(C+\lambda_{2}\right)^{2}}-E \\ 0 & 0 & \lambda_{6} \ln \left(\frac{\lambda_{7}}{C}\right)-\lambda_{6}-\lambda_{8}-E\end{array}\right]$

For $\mathrm{T}, \mathrm{E}$, and $\mathrm{C}$ if we plug a set of equilibrium values in matrix $\mathrm{J}$ then matrix $\mathrm{J}$ will show the linearization of the system of deferential equations about the particular equilibrium solution. By the Hartman-Grobman theorem, the nature of actual (nonlinear) system will be estimated by the nature of the linearized system close to the particular equilibrium solutions, considering the equilibrium solution is hyperbolic (that is eigenvalues of the matrix, real parts are not equal to zero). Now we will investigate both the equilibrium solution distinctly.

\subsection{Equilibrium 1}

Calculating $J$ at the trivial solution $E_{1}=(1,0,0)$ provide

$$
J\left(E_{1}\right)=\left[\begin{array}{ccc}
-1 & 0 & -\frac{\lambda_{1}}{\lambda_{2}} \\
0 & -\lambda_{5} & -\frac{\lambda_{3}}{\lambda_{2}} \\
0 & 0 & -\lambda_{6}-\lambda_{8}
\end{array}\right]
$$

The eigenvalues of the above matrix are $K_{1}=-1, K_{2}=-\lambda_{5}$ and $K_{3}=$ 
$-\lambda_{6}-\lambda_{8}$ are real numbers. Asymptotically stable for the equilibrium solution E1, so $K_{1}, K_{2}$ and $K_{3}$ are all negative.

\subsection{Equilibrium 2}

Here we investigate $E_{2}=(T, E, C)$ the equilibrium solution, when it exists. In this situation, by virtue of complexity of the system analytic approach to provide knowledge about eigenvalues, condition like Routh test e.g. provide transcendental equations, which may not be determined analytically [13]. Hence, oppositely, for a vast range of possible parameter values by sampling systematically, we investigate the eigenvalues over the ranges given in Table 1. Here we utilize an approach known as Latin hypercube sampling (LHS) to protect representative sampling. LHS is already known-technique for examine system which is more difficult to be controllable analytically and here likely huge uncertainty in the values of parameter which is utilized in the equations. The method includes numerically determining the equations repeatedly, utilizing randomly sampled values for entire parameters in the system. The samples are selected from the ranges of values are specified earlier and values of parameter are involved here, are selected to protect that all clinically sensible. Each range is divided into specified number of equi-probable intervals which are then plug in a random order, an any provided run a random value is chosen for which each one parameter from the appropriate interval. This protects the mixing of the intervals from which the random values are selected and provide higher confidence in the qualitative nature of the solutions against the uncertainty in values of parameter. The interval $(0,50000)$ that we sample for the equilibrium solutions for $T$, and $E$, and $(1,400000)$ is the interval that we sample for $C$. In LHS of 3000 run all of the eigenvalues were imaginary, or may be negative real, which bounded above by $-1.000076[1,13]$ for the entire real part. Thus, large ranges of $T, E$ and $C$ utilized to calculate the eigenvalues approximately provide acceptable confidence that the eigenvalues absolutely have negative real parts for the mentioned equilibrium solution, so we assume that here the solution is asymptotically stable. If $\frac{d_{c}}{r_{c}}<\ln \left(\frac{\gamma_{c} c_{\max }}{d_{n}}\right)$ (the parameter approximately in this situation), then the above system of Equation (12) have two asymptotically stable equilibria, $E_{1}$ and $E_{2}$ or else, the system has purely one equilibrium the trivial (healthy) say $E_{1}$ and this equilibrium is asymptotically stable [14].

\section{REFERENCES}

[1] Moore, H., Natasha, K.L. 2004. A mathematical model of chronic Myelogeous leukemia (CML) and $T$ cell interaction. Journal of Theoretical Biology, 227, 513-523.

[2] Khuri, S.A. 2001. A Laplace decomposition algorithm applied to a class of nonlinear deferential equations. Journal of Applied Mathematics,

\section{$1(4), 141-155$}

[3] Madani, M., Fathizadeh, M. 2010. Homotopy pertrubation algorithm using Laplace Transformation. Nonlinear Science Letters A, 1 (3), 263-267.

[4] Lu, J., Chen, G., Cheng, D., Celikovsky, S. 2002. Bridge the gap between the Lorenz system and the Chen system. International Journal of Bifurcation and Chaos, 12 (12), 2017-2926.

[5] Nanda, S., Moore, H., Lenhart, S. 2007. Optimal Control of treatment in a mathematical model of Chronic myelogenous Leukemia. Mathematical Bioscience, 210, 143-156.

[6] Ongun, M.Y. 2011. The Laplace Adomian Decomposition Method for solving a model for HIV infec-tion of $C D_{4}^{+} T$ cells. Mathematical and Computer Modeling, 53, 597-603.

[7] Adomian, G. 1991. A review of the decomposition method and some recent results for nonlinear equations. Applied Mathematics and Computation, 21 (5), 101-127. MR 92h: 00002b. Zbl 0732.35003.

[8] Khan, Y., Faraz, N. 2011. Application of modified Laplace decomposition method for solving boundray layer equation. Journal of King Saud University - Science, 23, 115-119.

[9] Gonzale-Gaxiola, 0. 2017. The Laplace Adomian decomposition method applied to the Kundu-Eckhaus equation. International Journal of Mathematics And its Applications, 1-12. ISSN: 2347-1557.

[10] Bellomo, N., Monaco, R.A. 1985. Comparison between Adomian decomposition method and pertrubation techniques for nonlinear random deferential equations. Journal of Mathematical Analysis and Applications, 110, 495-502.

[11] Adomian, G. 1994. Solving Frontier Problems of physics: The decomposition Method. Kluwer Academic Publication, Boston.

[12] Blower, S., Dowlatabadi, H. 1994. Sensitivity and uncertainty analysis of complex models of disease transmission: an HIV model as example. International Statistical Review, 2, 229-243.

[13] Borrelli, R., Coleman, C. 1998. Deferential Equations: A modeling Perspective. Willey, New York.

[14] Fokas, A.S., Keller, J.B., Clarkson, B.D., 1991. A mathematical moel of granulocytopoiesis and chronic myelogenous leukemia. Cancer Research, 51 (8), 2084-2091 